\title{
A convenient and accurate parallel Input/Output USB device for E-Prime
}

\author{
Rosario Canto • Ilaria Bufalari • Alessandro D'Ausilio
}

Published online: 4 December 2010

(C) Psychonomic Society, Inc. 2010

\begin{abstract}
Psychological and neurophysiological experiments require the accurate control of timing and synchrony for Input/Output signals. For instance, a typical EventRelated Potential (ERP) study requires an extremely accurate synchronization of stimulus delivery with recordings. This is typically done via computer software such as E-Prime, and fast communications are typically assured by the Parallel Port (PP). However, the PP is an old and disappearing technology that, for example, is no longer available on portable computers. Here we propose a convenient USB device enabling parallel I/O capabilities. We tested this device against the PP on both a desktop and a laptop machine in different stress tests. Our data demonstrate the accuracy of our system, which suggests that it may be a good substitute for the PP with E-Prime.
\end{abstract}

Keywords Low-cost I/O device $\cdot$ E-Prime $\cdot$ Timing accuracy

The authors would like to disclose their commercial interest on the device tested in the present article.

R. Canto $(\square) \cdot$ I. Bufalari $\cdot$ A. D'Ausilio $(\square)$

DSBTA - Section of Human Physiology, University of Ferrara,

Ferrara, Italy

e-mail: rosario.canto@unife.it

e-mail: alessandro.dausilio@iit.it

R. Canto $\cdot$ I. Bufalari $\cdot$ A. D'Ausilio

RBCS - Robotics, Brain and Cognitive Sciences Department, IIT -

The Italian Institute of Technology,

Genoa, Italy

\section{Introduction}

Most psychological and neurophysiological experiments require the accurate control of stimulus generation, response recording and external device synchronization. Such operations are typically performed via custom-made or public software. For instance, the experimenter may want to measure different aspects of human behavior (measured with button press, force sensors, optical or touch sensors, accelerometers, etc.) in association with other neurophysiological indices (as measured by other external equipment, e.g., electroencephalography, functional magnetic resonance imaging, transcranial magnetic stimulation, etc.). These kinds of experiments are based on the general assumption of correlating brain states with measurable behavioral variables (Gazzaniga, Ivry, \& Mangun, 2002). Therefore, computer controlled experiments require accuracy and consistency when dealing with timing and synchrony among separate machines.

In recent years, both commercial and open-source software have been proposed for Experimental Control and Management (ECM) such as E-Prime, Presentation, PsyScope, or the PsychToolbox for MatLab, among others. Previous studies suggest that new generation operating systems are not optimized for timing accuracy (Chambers \& Brown, 2003; MacInnes \& Taylor, 2001). Thus, the advantage of using ECM software is that they are designed for this specific purpose. Indeed, they often incorporate low level routines and set the highest priority to them. In fact, these software environments promise millisecond precision in these operations and are productively used in thousands of labs around the world. However, it is always advisable to check, on a case-by-case basis, the timing accuracy of experimental scripts and machines (Plant, Hammond, \& Turner, 2004). 
Synchronous recordings are typically achieved by relying on a master computer controlling the experimental work-flow and by sending triggers to other computers or external equipment. These triggers are then used by slave systems for synchronization, starting acquisitions or the generation of further commands. These basic I/O tasks are generally handled via the Parallel Port (PP). This port has proven to be a reliable and easy to use solution in psychological and neurophysiological settings (Stewart, 2006; Voss, Leonhart, \& Stahl, 2007).

Nevertheless, the PP is an end of life technology. The PP was originally developed to control printers. Nowadays it has been replaced by other ports such as the Universal Serial Bus (USB). Most recent motherboards do not even include the PP and one must resort to Peripheral Component Interconnect (PCI) or PCI express external boards. However, different boards from different manufacturers may have different timing performance (http://www.pstnet.com/eprimeport.cfm) and one must pay particular attention when building a new experimental setup. Moreover, laptops no longer include a PP. Until some years ago, laptops were not a suitable experimental platform due to an inherent lack of performance. However, a new generation of portable computers offers quite enough power to run most kinds of experiments and in some cases they might even be the platform of choice. Indeed, experiments run outside usual lab settings (such as hospitals, schools, subject's home, etc.) may require a portable setup.

Therefore, the current solution for those people requiring a portable experimental setup is either the use of Personal Computer Memory Card International Association (PCMCIA) adapters or a move to USB technology. PCMCIA - PP adapters, however, proved extremely unreliable in the time domain (http://www.pstnet.com/eprimeport.cfm) and therefore may not be a viable alternative. On the other hand, USB protocols unlike the PP are based on periodic polling. The host computer periodically queries input devices for state changes. Detection of any event is quantized in time with an interval that is typically fixed at $8 \mathrm{~ms}$. Moreover, additional uncertain delays could arise due to variability in the latency between event detection, handling by the operating system, as well as registration by the ECM program. Therefore, a current USB-based solution does not guarantee the necessary timing accuracy.

In order to solve this issue, we present a new commercial USB device provided with an onboard processor and memory buffer. This device can be used as a virtual PP in E-Prime, keeping control of event timing with sub-millisecond accuracy. The device has I/O capability and we offer a set of software tools that allow for complete integration in the E-Prime environment (Fig. 1). We tested our USB device along side the PP in several timing tests on a last-generation desktop computer to verify the reliability of our product against the gold-standard in the field (PP). We also ran a typical EEG Event-Related Potential (ERP) E-Prime script on both a desktop and a laptop, to test the reliability of our product in normal lab usage. Timing in all tests was verified via an external A/D board with an independent high-precision clock.

\section{Methods}

\section{Device description}

The device includes eight Transistor-Transistor Logic (TTL) input/output ( $\mathrm{I} / \mathrm{O})$ ports $(0$ to $+5 \mathrm{~V})$. Each port has a temporal resolution of $50 \mathrm{~ns}$ and the complete output voltage transition from Ground (GND) to $5 \mathrm{~V}$ takes approximately $400 \mu \mathrm{s}$. The device is controlled via a FT245RL USB to Parallel First-In-First-Out (FIFO) chip from Future Technology Devices International Limited (FTDI; www.ftdichip.com). This FTDI chip includes a $12-\mathrm{MHz}$ microprocessor. A Windows compatible .dll enables communication with the device. Thanks to the dedicated drivers provided by FTDI, port status from the device can be read or written at up to 1-M baud rate, or $14,745,600$ times per second, making polling delays negligible.

\section{E-Prime integration}

E-Prime v.2 integration was reached via a custom made Package, built with E-Prime Professional edition (v.2). Compatibility with E-Prime v.1 scripts is achieved by
Fig. 1 Panel A shows an upper side view of the device. Eight channels are present, each one with a LED showing $\mathrm{I} / \mathrm{O}$ activity. Panel B shows the USB port to be connected to the host computer. I/O connectivity is via standard Bayonet Neill-Concelman connectors (BNC)
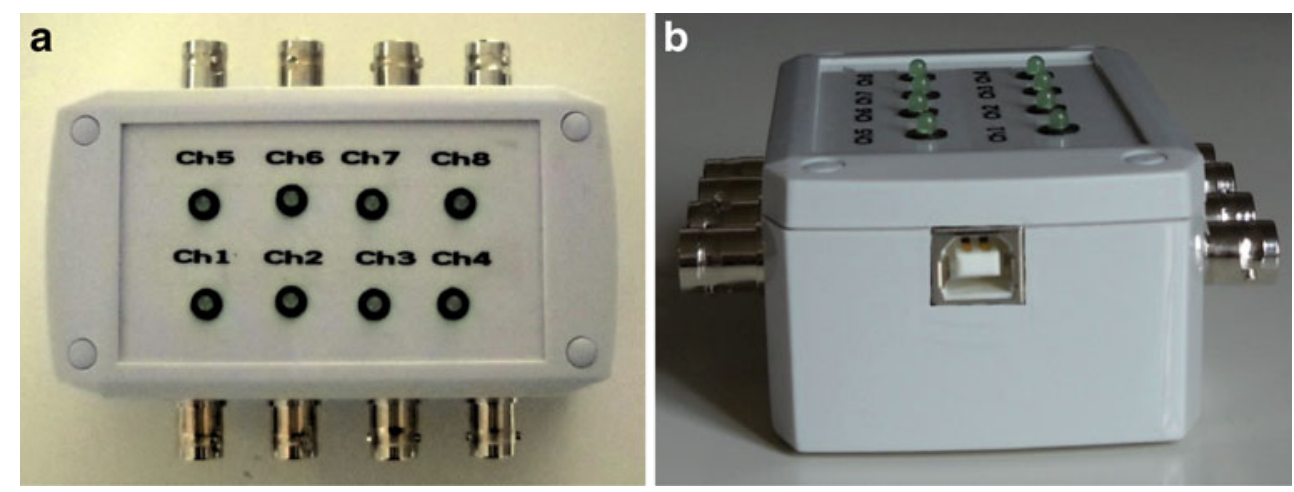
adding few lines of code in the script editor (procedures are described here: http://web.unife.it/progetti/neurolab/upf/). After loading our custom Package in E-Prime v.2, one can send or receive a TTL pulse over a given port as typically done to command a PP pin read or write. The read command is "readUPF". The write command is "writeUPF". The I/O port definition is performed by the "setChannel" command (further details and examples can be found at: http://web.unife.it/progetti/neurolab/upf/).

Test machine description

The desktop machine was based on an Intel Core Quad Q8200, 2.3 MHz, 2 GB RAM $1066 \mathrm{MHz}$, motherboard ASUS P5QL-E with a 1066-MHz bus, a 7200-rpm hard-disk with 32-MB Cache, with Windows XP and Service Pack 3. The parallel port was a Manhattan PCI Card including a MosChip 9865 chipset. The laptop machine was a Windows XP (Service Pack 3) ASUS Pentium M 740PCI Express with an ATI X700 128-MB DDR graphic card, $512 \mathrm{MB}$ of DDR2 $400-\mathrm{MHz}(512 \times 1)$ ram and an 80-GB 4200-rpm hard-disk.

\section{Timing control}

As shown in several papers, exact timing should always be controlled via external equipment with an independent high-precision clock such as external A/D boards or digital oscilloscopes (Chambers \& Brown, 2003; De Clercq, Crombez, Buysse, \& Roeyers, 2003; Plant, Hammond, \& Whitehouse, 2002). Here we used a CED1401Plus (Cambridge Electronics, UK) and Signal (version 3.1) software to store data for offline analyses. Data was acquired at $100 \mathrm{Khz}$.

Tests

Test1 was aimed at measuring the accuracy of $\mathrm{I} / \mathrm{O}$ capabilities of our device and comparing its accuracy with the PP performance. We made use of a test script downloaded from the E-Prime website (http://www.pstnet.com/ eprimeport.cfm) specifically to stress $\mathrm{I} / \mathrm{O}$ performance of the PP on the desktop computer. This script consists of the generation of square pulses lasting $100 \mathrm{~ms}$ with an interval of $50 \mathrm{~ms}$. Here we generated this sequence of square pulses from both the PP and the USB in parallel (500 pulses). We recorded both outputs on two separate A/D board analog channels.

Test2 is the same as Test1 run on a laptop. Here we had no PP signal recorded by the A/D board and we only verified that pulse length was kept constant and was correct (500 repetitions).

Test3 was an E-Prime simulation of a typical Event Related Potential (ERP) experiment with the desktop system. As typically done in ERP research, the E-Prime script generates both stimuli and sends synchronous triggers to the electroencephalographic (EEG) amplifiers. Here the script generated static pictures on a CRT monitor lasting $100 \mathrm{~ms}$ as well as a synchronous 10-ms TTL output pulse. The TTL was delivered independently from both the PP and USB at the onset of the image as in typical recording of visual ERPs. In these kinds of experiments, EEG traces have to be marked with external signals at the exact onset of the visual stimuli on the screen. However, since we cannot fully trust E-Prime timing, the real synchrony between the TTLs and onscreen picture appearance must be controlled externally. Therefore, synchrony was checked by using an on screen photodiode, placed on the upper-left corner of a CRT monitor set at $60-\mathrm{Hz}$ refresh rate. We fed the USB and PP output pulses, together with the photodiode signal, to the $\mathrm{A} / \mathrm{D}$ board on different analog inputs. We measured 500 repetitions on three $\mathrm{A} / \mathrm{D}$ channels (PP, USB, photodiode).

Test 4 is the same as Test 3 run on the laptop, and hence without the PP. Here we simulated an ERP study run on a laptop connected to the same CRT screen. Specifically we recorded the synchrony between the photodiode signal and the USB output (500 repetitions).

Analysis

All data recorded by the A/D board was exported to ASCII format and imported into the MatLab environment. We extracted, for each test, descriptive statistics such as the mean, standard deviation, maximum value and minimum value. For those tests run on the desktop computer we also compared the results of the PP and of the USB device.

\section{Results}

Test1 and Test2 explore whether the USB device can deliver a reliable TTL signal as typically done with the PP. In Test 1 we checked if output signals generated by the PP and this new device were synchronous and the duration was accurate on a desktop computer. Test 2 is run on a laptop and tests the USB device output timing performance. Tables 1 and 2 shows the results of Test 1 and Test2. The mean onset difference between the PP and the USB, as well as the pulse length in both the PP and USB device, is shown together with the USB pulse length on the laptop platform. All measures include standard deviations (SD), max values, min values and delta between max and min. On average we find that on a desktop machine PP and USB and USB on a laptop yielded similar and precise results regarding pulse length but more importantly the SD was in all cases below $1 \mathrm{~ms}$ (Desktop: PP $=0.000039 \mathrm{~ms}$, USB $=0.000147 \mathrm{~ms}$; 
Table 1 Results of Test 1 and Test2. These tests verify the accuracy of generating TTL pulses via a publicly available E-Prime script (see text) on both machines. Mean pulse duration, standard deviation (SD), range of variation (max, min, delta) are shown. All numbers are units in seconds. PP and USB represent the pulse length generated via the two ports. E-Prime generated a $100-\mathrm{ms}$ pulse, thus a small error around $100 \mathrm{~ms}$ represents a good performance. USB-PP shows the pulse onset asynchrony between the two ports. Mean onset difference, standard deviation (SD) and range of variation (max, min, delta) are shown

\begin{tabular}{|c|c|c|c|c|c|c|}
\hline PULSE test & & Mean & SD & $\max$ & $\min$ & delta \\
\hline \multicolumn{7}{|l|}{ LENGTH } \\
\hline \multirow[t]{2}{*}{ Desktop } & $P P$ & 0.099998 & 0.000039 & 0.100080 & 0.099260 & 0.000820 \\
\hline & $U S B$ & 0.100985 & 0.000147 & 0.101340 & 0.100000 & 0.001340 \\
\hline Laptop & $U S B$ & 0.100371 & 0.000440 & 0.101000 & 0.099650 & 0.001350 \\
\hline \multicolumn{7}{|l|}{ ONSET } \\
\hline Desktop & $P P-U S B$ & -0.000594 & 0.000173 & -0.000180 & -0.000830 & 0.000650 \\
\hline
\end{tabular}

Laptop: USB $=0.00044)$. As a confirmation, if we look at the trial by trial difference between PP and USB on the desktop only, we can see that the average asynchrony is less than $1 \mathrm{~ms}(-0.000594 \mathrm{~ms})$ and the SD is less than $1 \mathrm{~ms}$ (0.000173 ms).

Test 3 and Test 4 simulated the needs occurring in typical ERP research. Generally speaking, ERP research requires the application of markers on the EEG traces at the onset of externally driven sensory stimulations. Therefore, the synchrony between a TTL signal and the onset of a visual stimulus on the screen is critical. Table 1 shows the results of Test 3 and Test 4 . Here, the timing of visual stimuli appearance as well as the PP and USB triggers was controlled by E-Prime. Therefore, we verified the mean onset difference between the PP, USB, and photodiode on the desktop, or between USB and photodiode on the laptop platform. Here we find that the appearance of the visual stimulus on the CRT monitor and the PP or USB onset asynchrony was less than $1 \mathrm{~ms}(\mathrm{PP}=0.0001 \mathrm{~ms}$; USB $=$ $0.0004 \mathrm{~ms}$ ). Test4, which was run only on the laptop, showed an onset asynchrony between the USB and visual stimulus that is a bit larger $(0.002999 \mathrm{~ms})$, however the SD was below $1 \mathrm{~ms}(0.000342 \mathrm{~ms})$. Often the $\mathrm{SD}$ is more important than absolute values. Indeed, large but constant errors are usually better than small but varying delays. In fact, a systematic error with negligible SD may be easily corrected via the addition of an offset. However, one should always test experimental scripts beforehand via external A/ $\mathrm{D}$ boards, since mean error and SD may be very different on different computers.

\section{Discussion}

Experimental timing and event synchronization is a critical component of every psychological and neurophysiological experiment. Specific hardware and software has been proposed and marketed to achieve millisecond precision and the PP is almost the only way to let computers, devices, and equipment communicate efficiently. Unfortunately, the PP is disappearing, and is no longer available on portable computers. At the same time, portable systems are becoming the platform of choice at least in settings where flexibility and portability is required.

Here we propose a new low-cost USB device granting $\mathrm{I} / \mathrm{O}$ ability and the necessary temporal accuracy required for psychological and neuropsychological experiments. We also built specific software tools to interface our $\mathrm{I} / \mathrm{O}$ device with E-Prime, which is one of the most used commercial ECMs. We implemented several tests to verify the performance of this new device and the classic PP. Moreover, we ran a prototypical ERP experiment where temporal synchrony is extremely important. Our stress tests indicate that the $\mathrm{I} / \mathrm{O}$ device may be used in psychological
Table 2 Results of Test3 and Test4. These tests simulate a typical ERP study on both machines. USB-PP shows the pulse onset asynchrony between the two ports. Mean onset difference, standard deviation (SD) and range of variation ( $\max , \mathrm{min}$, delta) are shown. All numbers are units in seconds. PP and USB represent the synchronization error between each port separately and the on-screen appearance of the visual stimulus, as measured with the photodiode

\begin{tabular}{lllrrr}
\hline ERP tests & & Mean & SD & max & delta \\
\hline Desktop & USB-PP & 0.000527 & 0.000304 & 0.001060 & 0.00006 \\
& PP & 0.000093 & 0.000070 & 0.000310 & 0.001000 \\
& USB & -0.000434 & 0.000309 & 0.000120 & 0.00460 \\
Laptop & USB & 0.002999 & 0.000342 & 0.003720 & 0.00247 \\
\hline
\end{tabular}


and neurophysiological experiments where timing and external event synchrony is particularly important. Further information, and software download may be found at http:// web.unife.it/progetti/neurolab/upf/.

On the other hand, several USB or Serial devices have been proposed with quite limited functions. Most of them, in fact, can only acquire external button presses and therefore act only as response pads. Furthermore, these response pads rarely include an internal microprocessorcontrolled clock, memory buffer, and dedicated high-speed drivers. When no internal clock is included the burden of timing accuracy rests solely on the host computer ECM and drivers, with known limitations.

For example, both the response box from Engineering Solutions (www.response-box.com/tools.shtml) and the PST Serial Response Box from Psychology Software Tools (www.pstnet.com/products/SRBOX/default.htm) use the standard Serial port emulated over USB. However, apart from the lack of a dedicated hardware clock, these solutions are sub-optimal for three additional reasons: i. the serial port is rather un-common in recent computers; ii. serial emulation over USB usually has longer latencies than serial itself; iii. data reading is also vulnerable to timing uncertainty of the operating system. The PsyScope Button Box (http://psyscope. psy.cmu.edu/bbox/index.html) has its own clock, but according to its manual, it is still a work in progress. The RB Series Response Pads from Cedrus Corporation (www.cedrus.com/ support/rb_series) and the RTbox (Li, Liang, Kleiner, \& Lu, 2010) are the only devices using an internal clock to ensure timing accuracy. However, the Cedrus system, except for its response pad function, can receive only one TTL input for synchronization. The RTbox, instead, also has a photodiode and a sound input for stimulus onset synchronization ( $\mathrm{Li}$ et al., 2010). According to the RTbox website the newest version can handle I/O TTLs (http://lobes.usc.edu/RTbox/). However, this I/O communication seems to be still achieved via the serial port protocol and the authors offer only a MatLab integration.

A further advantage of the new device over other systems is its flexibility. The device is a general-purpose parallel I/O system that can acquire subjects' button presses (please check our website on how to build your own custom and cheap response pad) or external TTLs. This device can also generate multiple outputs to trigger or synchronize different external machines. Furthermore, complete flexibility is granted in E-Prime since it is possible to dynamically assign Input or Output properties to each channel, without additional programming or hardware modification. In short, the device can be used just as a normal PP in E-Prime. Moreover, the device offers several advantages since: $i$. it uses the USB port which is present in recent computers and will continue to be a standard in the future, with the adoption of the USB version 3 ; ii. it does no serial emulation over USB; iii. data reading is performed via dedicated drivers that provide microseconds precision. We conclude that that this device can offer the millisecond precision that is required for psychological and neurophysiological studies.

\section{References}

Chambers, C. D., \& Brown, M. (2003). Timing accuracy under microsoft windows revealed through external chronometry. Behavior Research Methods, Instruments, \& Computers, 35(1), 96-108.

De Clercq, A., Crombez, G., Buysse, A., \& Roeyers, H. (2003). A simple and sensitive method to measure timing accuracy. Behavior Research Methods, Instruments, \& Computers, 35(1), 109-115.

Gazzaniga, M. S., Ivry, R. B., \& Mangun, G. R. (2002). Cognitive neuroscience: The biology of the mind (2nd ed.). New York: W. W. Norton.

Li, X., Liang, Z., Kleiner, M., \& Lu, Z.-L. (2010). RTbox: A device for highly accurate response time measurements. Behavior Research Methods, 42(1), 212-225.

MacInnes, W. J., \& Taylor, T. L. (2001). Millisecond timing accuracy on PCs and Macs. Behavior Research Methods, Instruments, \& Computers, 33(2), 174-178.

Plant, R. R., Hammond, N., \& Turner, G. (2004). Self-validating presentation and response timing in cognitive paradigms: How and why? Behavior Research Methods, Instruments, \& Computers, 36(2), 291-303.

Plant, R. R., Hammond, N., \& Whitehouse, T. (2002). Toward an experimental timing standards lab: Benchmarking precision in the real world. Behavior Research Methods, Instruments, \& Computers, 34(2), 218-226.

Stewart, N. (2006). A PC parallel port button box provides millisecond response time accuracy under Linux. Behavioral Research Methods, 38(1), 170-173.

Voss, A., Leonhart, R., \& Stahl, C. (2007). How to make your own response boxes: A step-by-step guide for the construction of reliable and inexpensive parallel-port response pads from computer mice. Behavior Research Methods, 39(4), 797-801. 